\title{
Usefulness of combined handheld ultrasound and fluoroscopy-guided injection in adhesive capsulitis of the shoulder: A prospective, randomized single blind-pilot study
}

\author{
Ki Deok Park ${ }^{\mathrm{a}}$, Jeong Won Ryu ${ }^{\mathrm{b}}$, Kyoung Rai Cho ${ }^{\mathrm{c}}$, Yongbum Park ${ }^{\mathrm{d}, 1, *}$ and Wook-Jin Chung ${ }^{\mathrm{e}, \mathrm{f}, 1, *}$ \\ ${ }^{a}$ Department of Rehabilitation Medicine, Gil Medical Center, Gachon University College of Medicine, Incheon, \\ Korea \\ ${ }^{\mathrm{b}}$ Advanced Medical Technology Laboratory, Healcerion Co., Ltd., Seoul, Korea \\ ${ }^{\mathrm{c}}$ Department of Otorhinolaryngology-Head and Neck Surgery, Sanggye Paik Hospital, Inje University College of \\ Medicine, Seoul, Korea \\ ${ }^{\mathrm{d}}$ Department of Physical Medicine and Rehabilitation, Sanggye Paik Hospital, Inje University College of Medicine, \\ Seoul, Korea \\ ${ }^{\mathrm{e}}$ Department of Cardiovascular Medicine, Gil Medical Center, Gachon University College of Medicine, Incheon, \\ Korea \\ ${ }_{\mathrm{f}}^{\mathrm{f}}$ Gachon Cardiovascular Research Institute, Gachon University, Incheon, Korea
}

Received 11 June 2021

Accepted 20 October 2021

\begin{abstract}
.
BACKGROUND: Ultrasound is increasingly being utilized in the diagnosis and treatment of adhesive capsulitis.

OBJECTIVE: To compare the therapeutic effects and advantages of combined handheld ultrasound and fluoroscopy-guided intra-articular corticosteroid injection with those of conventional ultrasound-guided corticosteroid injection in adhesive capsulitis of the shoulder.

METHODS: A total of 39 patients diagnosed with adhesive capsulitis of the shoulder were randomly assigned into two groups. Group A patients $(n=19)$ underwent combined handheld ultrasound and fluoroscopy-guided corticosteroid injection and group B patients $(n=20)$ underwent conventional ultrasound-guided corticosteroid injection to the intra-articular space of the shoulder twice. Treatment efficacy was assessed at 2 and 6 weeks after the final injection, based on the verbal numeric pain scale, Shoulder Pain and Disability Index, and range of motion. Secondary outcome measures were the accuracy and procedure time.

RESULTS: Both injection methods were effective in the treatment of adhesive capsulitis. No significant differences in treatment efficacy and injection accuracy were observed between the two groups $(p>0.05)$.

CONCLUSIONS: This study showed no statistical differences in treatment efficacy between 2 groups. However, the combined use of ultrasound and fluoroscopy can increase the accuracy of injection compared with conventional ultrasound alone.
\end{abstract}

Keywords: Interventional ultrasound, frozen shoulder, intraarticular injection, fluoroscopy

\footnotetext{
${ }^{1}$ Yongbum Park and Wook-Jin Chung, contributed equally to this work as co-corresponding authors.

${ }^{*}$ Corresponding authors: Yongbum Park, Sanggye Paik Hospital, Inje University College of Medicine, 761-1, Sanggye-dong, Nowon-
}

gu, Seoul 139-707, Korea. E-mail: swc328@naver.com; Wook-Jin Chung, 21 Namdong-daero, 774-gil, Namdong-gu, Incheon 21565, Korea. E-mail: heart@gilhospital.com. 


\section{Introduction}

Adhesive capsulitis is one of the most common causes of shoulder pain and disabilities [1]. This condition is characterized by a hypertrophic synovial capsule, adhesions and contracture of the glenohumeral capsule, fibrosis, and inflammatory processes [2,3]. Painful shoulder stiffness can adversely affect daily life activities and ultimately reduce the quality of life [3].

Several treatment methods, such as corticosteroid injection, use of nonsteroidal anti-inflammatory drugs, and physical therapy, can be applied for treating adhesive capsulitis of the shoulder [2]. Intra-articular (IA) injection was reported to be effective in decreasing pain and increasing the shoulder range of motion (ROM) in patients with adhesive capsulitis [4-7].

Ultrasound (US) is increasingly being utilized in the diagnosis and treatment of musculoskeletal diseases. A previous study has shown that US-guided IA corticosteroid injection has advantages over fluoroscopy (FL)guided capsular distension, including lack of radiation exposure, increased accuracy by viewing the expansion of joint spaces, and better convenience of use with similar therapeutic effects [8]. Although US has more advantages than FL, the US device is still bulky and difficult to transport and handle in health-care facilities.

Previous studies have reported the accuracy of USguided IA injection in the glenohumeral joint to be 90 93.3\% [9-11]. Extra-articular corticosteroid injections can lead to complications including soft-tissue atrophy, tendon degeneration or ruptures, and skin depigmentation [12]. Several studies have reported on combined FL and US-guided interventions for musculoskeletal pain treatment [13-15]. This combined method allows avoiding critical structures in the needle path while checking the progress of the needle in real time under US. In addition, by injecting a contrast media, it is possible to confirm whether the injection was performed at the correct site through the fluoroscopic image. Therefore, this combined method enables injecting with safety and accuracy. However, studies on this method with respect to musculoskeletal treatment are still lacking. Furthermore, using US and FL simultaneously requires a large space; mastering both methods can be difficult; and cost-effectiveness problems may exist.

A portable US device was developed in 1975, and a handheld US device was developed in the late 1990s. With continuous technological advancements, smaller and lighter devices that provide high-quality images became recently available [16]. A handheld device enables physicians to make instant assessments in or outside the hospital. It can reduce the patients' waiting time and enhance the clinicians' workflow [16]. Moreover, previous studies have shown the efficacy and convenience of use of handheld US systems, as well as their capability to detect some diseases [17].

The aim of this study was to compare the therapeutic effects and advantages of that combined FL and handheld US-guided and the conventional US-guided IA injection in order to verify the usefulness of that combined FL and handheld US-guided IA injection in the treatment of frozen shoulders.

\section{Materials and methods}

\subsection{Participants}

After obtaining institutional review board approval and clinical trial registration (GDIRB2017-113), 40 patients with frozen shoulder were enrolled into this a prospective, randomized single blind-pilot study. This study included patients who complained of shoulder pain and showed limitation of shoulder ROM from April to December 2017.

The diagnosis of adhesive capsulitis was made by two physiatrists based on history taking, physical examination, radiologic evaluation, and musculoskeletal US findings. The inclusion criteria were as follows: disease duration of 3-9 months and $\mathrm{a}>30^{\circ}$ reduction in passive ROM (pROM) in at least two of forward flexion, abduction, and external rotation of the affected shoulder compared with the contralateral side [8].

The exclusion criteria were as follows: US finding of a full-thickness tear of rotator cuff; hospitalization over the previous 6 months for a shoulder joint-related incident; a severe psychiatric problem; age $<19$ or $>70$ years; a plain radiographic finding of significant glenohumeral joint arthritis; systematic rheumatic disease; a neurologic disorder, such as cervical radiculopathy or stroke; a bleeding tendency; use of anticoagulants; diabetes mellitus (associated with adverse effects of corticosteroids); history of adverse reactions of local anesthetics; presence or suspicion of infection; or use of any drug (other than acetaminophen) during treatment or follow-up that might affect outcomes [8-10].

\subsection{Randomization of participants}

After the eligibility assessment, initial evaluation was performed. Participants were randomly assigned into one of 2 groups using a computer-generated randomization table. 


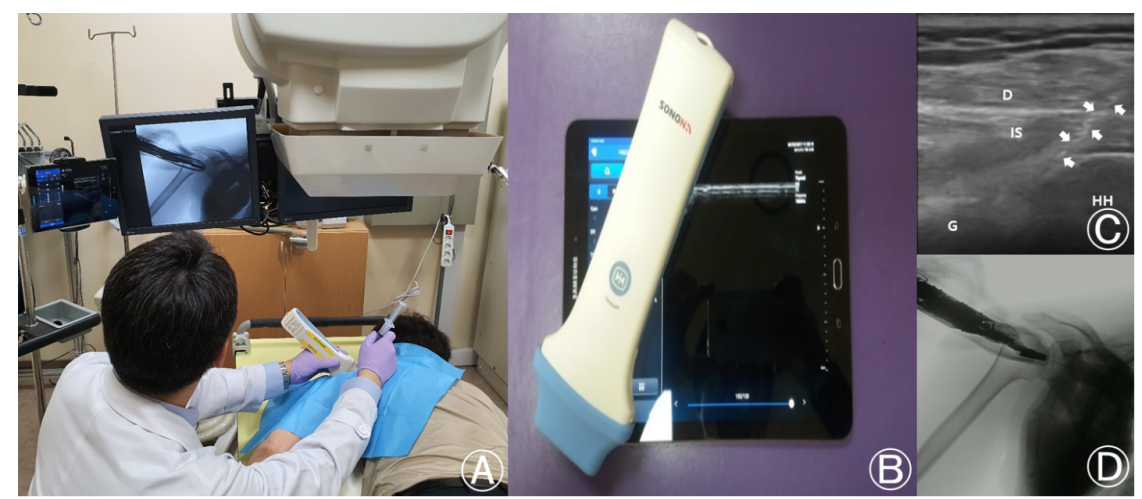

Fig. 1. Combined fluoroscopy (FL) and handheld ultrasound (US)-guided intra-articular (IA) injection technique. (A) Combined FL and handheld US equipment set. (B) Handheld US equipment set. (C) Image of the handheld US on the shoulder. D, deltoid; IS, infraspinatus; G, glenoid; HH, humeral head. Arrows indicate the needle. (D) Image of the FL with the handheld US equipment on the shoulder.

For group A, portable US (SONON 300L ${ }^{\circledR}$; Healcerion, Seoul, Korea) and FL (VersiFlex VISTA; HITACHI, Tokyo, Japan) devices were used during IA injection and for follow-up evaluation in the clinic room. The frequencies of the probes were 5.75 and $10 \mathrm{MHz}$, respectively. For group B, a conventional US device (LOGIQ S7 Expert ${ }^{\circledR}$; General Electric, Boston, MA, USA) was used in the US room.

The frequencies of the linear probes were between 5 and $15 \mathrm{MHz}$. Patients in groups A and B were administered the same mixture containing $0.5 \%$ lidocaine $(7 \mathrm{ml})$, triamcinolone $20 \mathrm{mg}(1 \mathrm{ml})$, and nonionic contrast medium (Omnipaque 300; GE Healthcare, Shanghai, USA; 2 mL).

\subsection{Combined FL and handheld US-guided IA injection technique}

All US examinations of the shoulder and combined FL and handheld US-guided IA injection procedures were performed by one physician (K.D. Park) with > 12 years' experience.

During the injection, the patients were placed on the $\mathrm{C}$-arm table in the prone position with their upper arm adducted and internally rotated. The posterior of the acromion was palpated, and the injection site was $1 \mathrm{~cm}$ inferior and $1 \mathrm{~cm}$ medial to the acromion. Under FL and handheld US guidance, injection with a posterolateral approach was performed using a 25-gauge, 2.5 inch needle (Fig. 1) [12,18]. All injections were made after ensuring with US that the needle tip was properly located in the IA space and finally checking the contrast medium in the joint cavity with FL.

\subsection{US-guided IA injection techniques}

During the injection, the patients were in a sitting position with their arm adducted and internally rotated. The posterior of the acromion was palpated, and the injection site was $1 \mathrm{~cm}$ inferior and $1 \mathrm{~cm}$ medial to the acromion. After needle entry, injection was performed toward the coracoid process. Under US guidance, injection with a posterolateral approach was performed using a 25-gauge, 2.5 inch needle. All injections were made after ensuring with US that the needle tip was properly located in the IA space. The accuracy of injection into the IA space was checked during the procedure, and joint capsule distension was assessed in appropriate cases (Fig. 2) [18].

The participants in both groups were asked to limit shoulder motion for at least $10 \mathrm{~min}$ after injection to allow the localization of the injected drugs in the articular capsule. All participants were prescribed a simple exercise program comprising pendulum exercise and scapular setting (isometric scapular retraction). Additionally, acetaminophen or ice massage was permitted in patients with pain at the injection site. The first IA injection was immediately performed after the diagnosis of adhesive capsulitis. After 2 weeks, a second IA injection was performed. The injection frequency was identical for groups A and B.

\subsection{Evaluation of accuracy, treatment effect and procedure time}

The accuracy of combined US and FL-guided injection can be immediately confirmed on the $\mathrm{C}$-arm image after contrast injection. For US-guided injection into the IA space, the accuracy was assessed using shoulder radiography after the injection. If contrast medium was observed in the glenohumeral joint space, the injection was considered to be accurate (Fig. 3). 


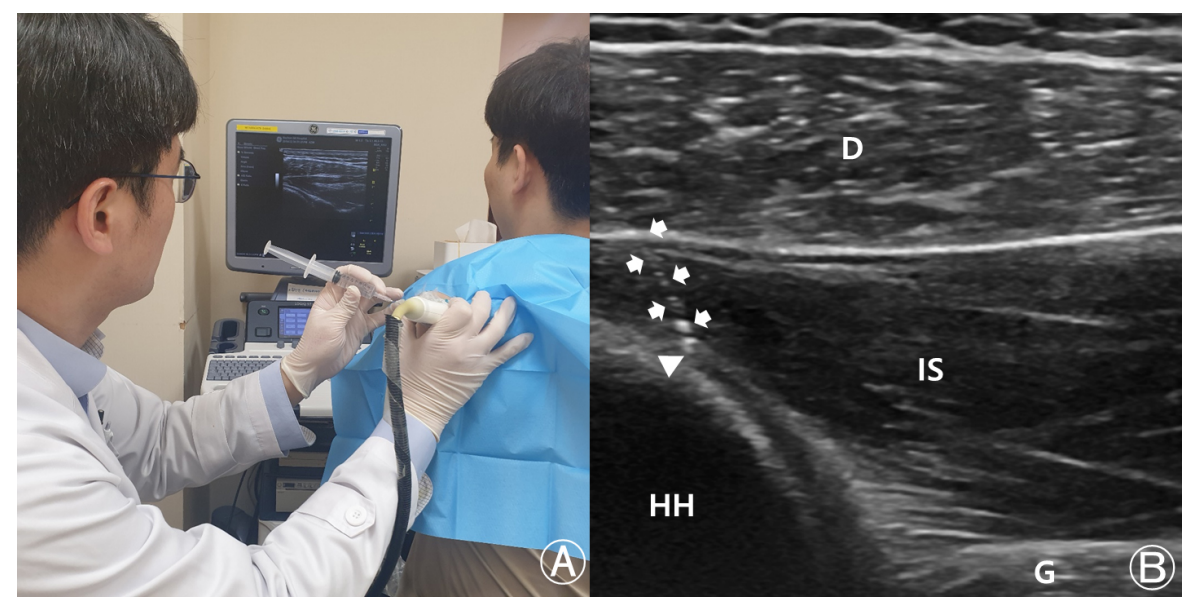

Fig. 2. Ultrasound (US)-guided intra-articular (IA) injection techniques. (A) US-guided IA injection with the posterolateral approach. (B) Image of the US on the shoulder. D, deltoid; IS, infraspinatus; G, glenoid; HH, humeral head. Arrows indicate the needle and arrowhead indicates the needle tip in the IA space.

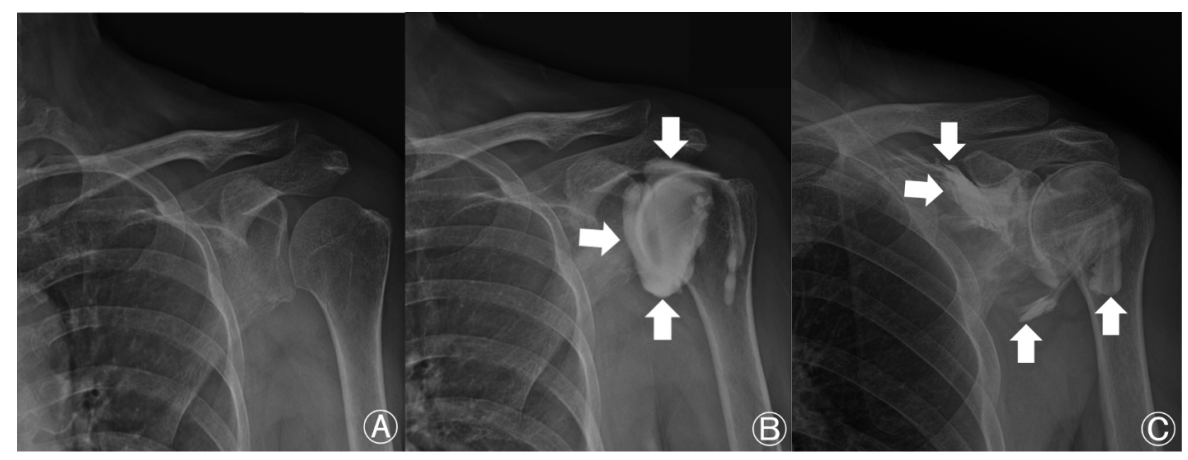

Fig. 3. Shoulder posterior-anterior view. (A) Before intra-articular (IA) steroid injection. (B) After IA corticosteroid injection. (C) Leakage of contrast medium to outside the articular space. Arrows indicate the contrast medium.

The evaluation of the treatment effects of IA injection was completed a total of three times: before the first injection and at 2 and 6 weeks after the second injection. Verbal numeric pain scale (VNS), Shoulder Pain and Disability Index (SPADI), and pROM were assessed during treatment efficacy evaluation. All assessments were performed by one evaluator who was unaware of patient grouping under supervision.

SPADI is a self-administered assessment tool that measures pain and disability related to shoulder disease [8]. It consists of five pain and eight disability items, each measured on a visual analogue scale. The pain and disability subscales are calculated as the mean of the corresponding items on a 0-100 scale, with the highest score indicating the most severe pain and disability. The total score is calculated by summing the pain and disability subscale scores. SPADI has been used in previous randomized trials investigating treatment effects in populations with frozen shoul- der [19-21]. The psychometric properties of the index have been tested in patients with shoulder problems, and the index has been shown to have acceptable validity for group comparisons [8].

The VNS is an 11-point scale ranging from 0 ("no pain") to 10 ("the worst pain possible) [8]. For the VNS evaluation, the patients were asked to rate their pain on a scale from 0 to 10 ( 0 and 10 represented "no pain" and "the worst pain possible," respectively), in whole numbers with 11 integers including zero [8].

ROM was measured by physicians with the patient in the supine position. A physician assisted the patient to achieve maximum passive motion, and another physician measured pROM values in three directions (forward flexion and abduction from neutral position and external rotation from $45^{\circ}$ abduction postion) using a Dualer IQ inclinometer (JTECH Medical, Salt Lake City, UT, USA). 
Table 1

Characteristics of the participants

\begin{tabular}{lccc}
\hline & Group A & Group B & $p$-value \\
\hline Number & 19 & 20 & \\
Sex & M2/F17 & M4/F16 & \\
Age (years) & $57.90 \pm 9.73$ & $55.65 \pm 7.29$ & 0.418 \\
Duration (months) & $6.48 \pm 3.44$ & $7.85 \pm 2.25$ & 0.148 \\
BMI $\left(\mathrm{kg} / \mathrm{m}^{2}\right)$ & $23.30 \pm 2.73$ & $22.06 \pm 2.82$ & 0.093 \\
Lesion side (R/L) & $8 / 11$ & $11 / 9$ & 0.434 \\
\hline
\end{tabular}

Values are mean \pm SD. Group A: combined handheld ultrasoundguided and fluoroscopy-guided intra-articular injection group. Group B: conventional ultrasound-guided intra-articular injection group. BMI, body mass index; F, female; L, left; M, male; R, right.

Procedure time was defined as the time interval between the point of contact of the US probe with the patient's skin and the completion of injection.

\subsection{Statistical analysis}

The purpose of this pilot study is to compare the therapeutic effects, advantages, and the feasibility for a further large-scale clinical trial of combined handheld ultrasound and fluoroscopy-guided intra-articular corticosteroid injection with those of conventional ultrasound guided steroid injection in adhesive capsulitis of the shoulder We did not find a suitable reference to calculate the sample size, but a minimum sample size of the clinical trial with 20 patients per group is used instead [22].

An independent t-test was used to compare differences in participant characteristics and procedure time between the two groups. Data acquired at the initial diagnosis of adhesive capsulitis, and those obtained at 2 and 6 weeks after the second injection, were used for comparisons between the two groups. Comparison of VNS score, ROM, and SPADI between the two groups was performed using a normality test of the ShapiroWilk test and a two-way repeated-measures analysis of variance. A p-value of $<0.05$ was set to indicate statistical significance. Statistical analysis was performed using IBM SPSS 18. (IBM Corp., Armonk, NY, USA).

\section{Results}

\subsection{General characteristics of patients}

Initially, 40 participants were randomly assigned and divided into two groups. A total of 20 participants were assigned into group A and the other 20 participants were assigned into group B. However, one of the participants in group A had a failed follow-up (Fig. 4). Finally, group
A was composed of 19 patients ( 2 men, 17 women) with a mean age of 57.90 years. The mean duration of disease among group A patients was 6.48 months. Group B was composed of 20 patients ( 4 men, 16 women) with a mean age of 55.65 years. Among the patients of this group, the mean disease duration was 7.85 months. No significant differences in the general patient characteristics were observed between the two groups $(p>$ 0.05) (Table 1).

\subsection{Change in pain severity according to VNS, SPADI and ROM in passive movements}

The VNS score before IA corticosteroid injection was $7.08 \pm 1.85$ in group A and $6.96 \pm 0.74$ in group $\mathrm{B}$, with no significant difference. At 2 and 6 weeks after the procedure, the VNS scores were decreased, with no significant difference $(2.41 \pm 1.21$ vs. $2.88 \pm 0.68$, $2.47 \pm 1.65$ vs. $2.52 \pm 0.66$ ). The VNS score between before injection and at 2 weeks after injection, and that between before injection and at 6 weeks after injection were significantly decreased in each group. However, no significant differences were observed between the two groups $(p>0.05)$ (Table 2).

The SPADI scores before injection were $60.23 \pm$ 11.40 and $64.52 \pm 10.07$ in groups $\mathrm{A}$ and $\mathrm{B}$, respectively. At 2 weeks after the final injection, the SPADI scores were $27.24 \pm 9.89$ and $30.23 \pm 8.96$, respectively. After 6 eeks, the SPADI scores were $26.42 \pm$ 8.82 and $28.35 \pm 7.96$ for groups A and B, respectively. Significant reductions in SPADI scores were observed after corticosteroid injection in both groups $(p<0.05)$. However, no significant differences were observed between the two groups $(p>0.05)$ (Table 2).

After the procedure, pROM showed improvements in both groups. Significant improvements in ROM for three types of movements were observed in both groups at 2 and 6 weeks after the procedure $(p<0.05)$. There were no significant differences between the two groups $(p>0.05)$ (Table 2).

\subsection{Accuracy and procedure time}

In all patients of group A, contrast medium was completely injected into the articular cavity, while three cases of leakage and injection outside the articular space were confirmed in group B (Fig. 3). No statistically significant difference in injection accuracy was observed between groups (19/19 (100\%) vs. 17/20 (85\%), $p>$ $0.05)$. No significant difference in the time needed for the injection procedure was observed between groups $(540.65 \pm 18.08$ vs. $480.32 \pm 25.73 \mathrm{sec}, p>0.05)$. 
Table 2

Comparison of the effect of intra-articular corticosteroid injections on VNS, SPADI, and pROM

\begin{tabular}{ccccc}
\hline & Group & $\begin{array}{c}\text { Before steroid } \\
\text { injections }\end{array}$ & $\begin{array}{c}\text { 2 wks. after } \\
2^{\text {nd }} \text { injection }\end{array}$ & $\begin{array}{c}6 \text { wks. after } \\
2^{\text {nd }} \text { injection }\end{array}$ \\
\hline VNS & A & $7.08 \pm 1.85$ & $2.41 \pm 1.21^{*}$ & $2.47 \pm 1.65^{*}$ \\
& B & $6.96 \pm 0.74$ & $2.88 \pm 0.68^{*}$ & $2.52 \pm 0.66^{*, \ddagger}$ \\
SPADI & A & $60.23 \pm 11.40$ & $27.24 \pm 9.89^{*}$ & $26.42 \pm 8.82^{*}$ \\
pROM & B & $64.52 \pm 10.07$ & $30.23 \pm 8.96^{*}$ & $28.35 \pm 7.96^{*, \ddagger}$ \\
FL & A & $131.58 \pm 17.00$ & $150.79 \pm 11.33^{*}$ & $154.74 \pm 11.48^{*}$, \\
& B & $126.25 \pm 10.50$ & $148.25 \pm 8.93^{*}$ & $151.50 \pm 10.65^{*}$ \\
AB & A & $106.32 \pm 28.96$ & $138.16 \pm 18.04^{*}$ & $139.74 \pm 13.43^{*}$ \\
& B & $100.25 \pm 11.06$ & $131.75 \pm 10.16^{*}$ & $133.25 \pm 2.33^{*}$ \\
& A & $48.95 \pm 23.43$ & $69.21 \pm 14.84^{*}$ & $70.53 \pm 13.11^{*}$ \\
\hline
\end{tabular}

Values are mean \pm SD. Group A: combined handheld ultrasound-guided and fluoroscopy-guided intra-articular injection group. Group B: conventional ultrasound-guided intra-articular injection group. AB, abduction; ER, external rotation; FL, flexion; pROM: passive range of motion; SPADI, Shoulder Pain and Disability Index; VNS, visual numeric scale; wks: weeks. ${ }^{*} p<0.05$ : before vs. after injection. ${ }^{\dagger} p<0.05$ : group A vs. group B. ${ }^{\ddagger} p<0.05: 2$ wks. after $2^{\text {nd }}$ injection vs. 6 wks. after $2^{\text {nd }}$ injection.

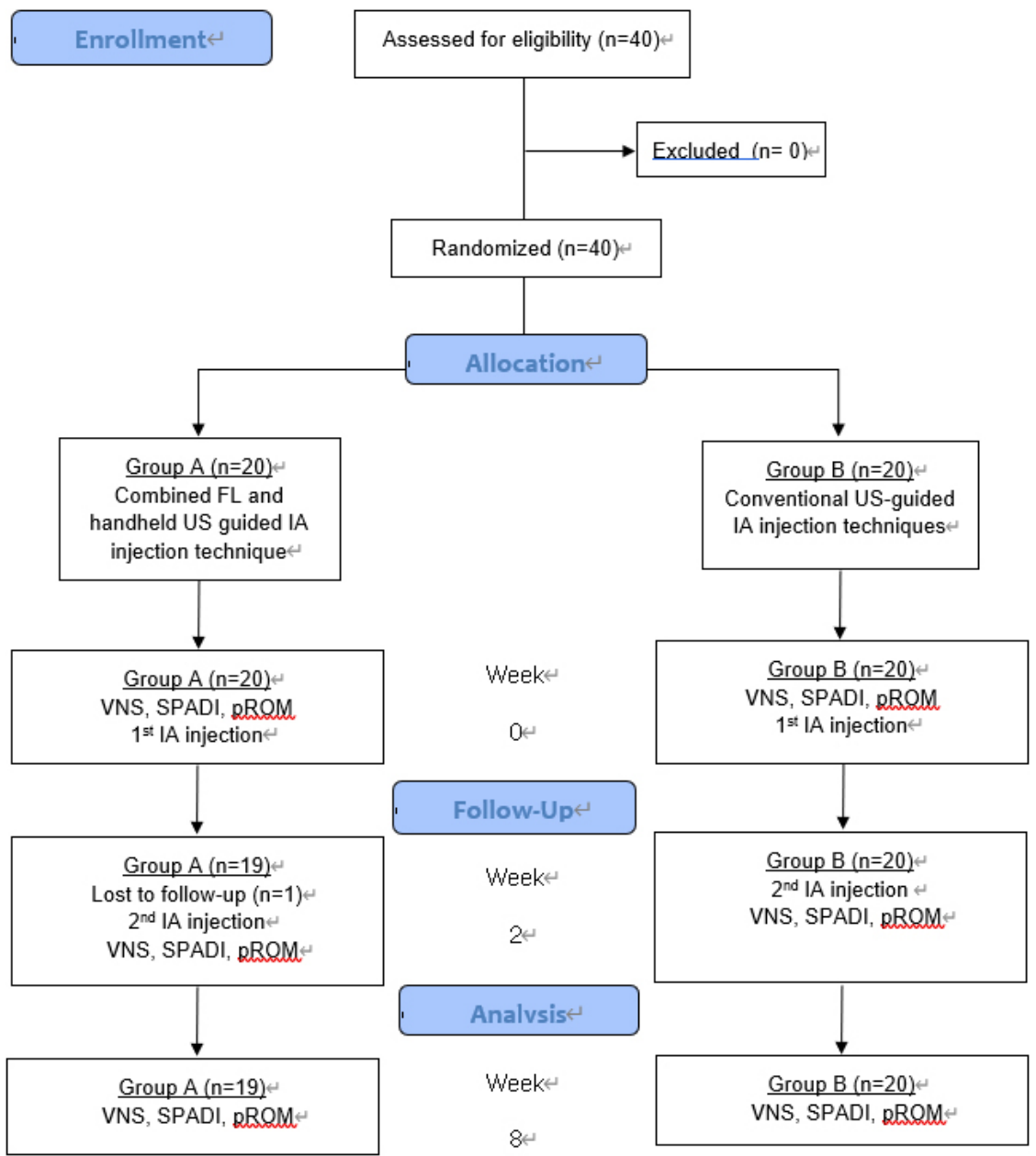

Fig. 4. Design and flow of participants in the study. FL, fluoroscopy; IA, intra-articular; US, ultrasound; VNS, verbal numeric pain scale; SPADI, shoulder pain and disability index; pROM, passive range of motion. 


\subsection{Complications}

No severe complications, such as vasovagal syncope, allergic reactions, steroid-associated infection, adipose tissue atrophy, or toxic reactions including dizziness, were observed after injections in both groups.

\section{Discussion}

Adhesive capsulitis commonly affects about $2-3 \%$ of the general population and up to $5 \%$ in certain populations [4,23,24]. Adhesive capsulitis can occur as a primary or secondary disorder. Primary adhesive capsulitis can occur spontaneously. Secondary adhesive capsulitis is often observed after shoulder joint fracture-dislocation, arm fracture, or other severe joint trauma [25].

IA corticosteroid injection has been reported to have greater efficacy than oral administration of steroids in the treatment of adhesive capsulitis [5]. Hettrich et al. [4] showed that IA corticosteroid injection is effective in decreasing fibromatosis and myofibroblast numbers in capsular tissue. Other studies have also reported improvements in pain and ROM after IA corticosteroid injection [6,7]. In IA corticosteroid injection, the accuracy of the injection is the key factor that determines the inflammation-reducing effect [26,27]. As accurate injection into the desired site is crucial for ensuring the therapeutic effect, guidance using several modalities can enhance the efficacy of corticosteroid injection treatment. US guidance is one of the modalities that can be utilized in IA corticosteroid injection. Previous studies have shown that US guidance can be utilized in the treatment of adhesive capsulitis of the shoulder $[1,8]$.

US is an excellent visualization tool that avoids vulnerable structures such as nerves and vessels during injection, whereas contrast FL can only detect when the tip of the needle is at the target point. It should be kept in mind that FL may not be able to detect that the needle has already traversed a vessel on its way to the target, whereas US can avoid this problem [28]. Therefore, if both methods are used simultaneously, patient safety and more accurate injection can be guaranteed than by injection with each method alone. Some studies on the use of the combined US and FL-guided injection method for detecting renal abscess, for kidney biopsy, and for the treatment of musculoskeletal disorders have been recently; however, data are still insufficient [29-32].

In US-guided IA shoulder injection with a posterior approach, the direction of the needle tip can be checked in real time. After the needle is positioned at the target point, the drug can be injected into the IA space; however, because a contrast agent is not used, it is difficult to directly check the accuracy of the injection into the joint cavity. Moreover, obese patients have a disadvantage in that it is difficult to check the needle tip position on the US image, and, when injected, the drug may flow out of the joint cavity, causing some adverse effects. The FL-guided injection method has the advantage of using a contrast agent to ensure the accurate injection of the drug into the joint cavity; however, it is costly, bulky, and exposes the patient and clinician to ionizing radiation. A risk of damage to the major structures in the path of needle progression also exist.

If the two methods are used simultaneously, critical structures can be avoided in the injection process, needles can be placed at the target point, and the accurate injection of the drug can be detected with contrast medium use. Furthermore, despite the risk of radiation exposure, the amount of irradiation may be smaller than when using FL alone. The disadvantages of using both methods simultaneously are that both the FL and US equipment are bulky. Thus, in this study, the spatial constraints were reduced through the use of handheld US.

Cases of injection failure in group B included not only a direct extra-articular space injection but also cases in which the nonionic contrast medium was visible in bursal layers because of drug leakage after an IA injection. In total, there were three failed cases: In all three cases, all injections were accurately performed on the targeted site, joint capsular distension was confirmed during injection and capsular rupture was not observed. These failures were primarily attributed to technical problems, not to patient factors. The causes of such failures were the following: (1) potential cases in which the position of needle in the course of injection could not be identified, such that the needle used for the procedure could be easily misaligned because of the narrow beam of US to the extent of $1 \mathrm{~mm}$, and (2) the needles may not have been fixed correctly because a freehand technique was used in this study.

Intraarticular corticosteroid injection failure such as extraarticular space leakage can cause temporary increases in pain, skin atrophy, depigmentation, and septic arthritis as well as deleterious effects on tendon degeneration and even tendon ruptures [33]. Although there were no statistical significance, three extraarticular leakage injection cases were observed in group B. Accurate intraarticular injection is essential to ensure safety of procedure, and this can be made by use of 
assistant modalities such as US. Studies have been reported about advantages and safety for combined US and FL guidance injection technique for the treatment of musculoskeletal pain through complementing each method's weakness [34,35].

Many previous studies have reported the applicability of handheld US in diagnosing diseases in many subfields of medicine. Tse et al. [16] compared the performance of a handheld US device against the conventional US device in terms of image quality in common abdominal pathologies. In their study, the handheld US device showed satisfactory imaging quality and proved to be useful in emergency screening or general screening of abdominal organ pathologies. In the field of musculoskeletal diseases, we expect that a handheld device would not be inferior to a conventional device and would be more convenient in diagnosing and treating both outpatients and inpatients. Moreover, we were set on the idea of using a handheld device for US guidance in the treatment of adhesive capsulitis, specifically with IA corticosteroid injection.

After injections, both groups showed significant improvements in pain severity and ROM. The differences in VNS and ROM between 2 and 6 weeks after the second injection were significantly lower than when they were compared with the initial assessment. However, the efficacy of treatment showed no significant differences between group A (combined handheld US and FL) and group B (conventional US). Moreover, in group A, the effect of flexion continued until 6 weeks after the last injection and was more effective than after 2 weeks. In group B, the effect continued for 6 weeks after injection, and the SPADI and VNS score were also significantly different between 2 and 6 weeks after injection. These results imply that both groups showed attenuated symptoms of adhesive capsulitis and that the corticosteroid injection treatment was effective, which suggests that handheld US under FL is not inferior to FL

The time consumed for the whole procedure was compared between groups $\mathrm{A}$ and $\mathrm{B}$. The whole injection procedure took about 9 min in group $A$ and about $8 \mathrm{~min}$ in group B. The combined method requires additional time to check the FL image after the injection; however, no statistical differences were observed. Although the combined method may take longer than US guidance alone, it is recommended as a safe method because it can help reduce the complications caused by leakage of drugs out of joint spaces through contrast medium detection and has lower radiation exposure than with using FL alone.

\subsection{Limitations}

One of the limitations of this study was the small sample size in each group. Only 19 and 20 patients were included in groups A and B, respectively. The short follow-up period was also a limitation. Subsequent studies with larger numbers of participants and with longer follow-up periods should be conducted to thoroughly evaluate the outcomes of US-guided IA steroid injection in the treatment of adhesive capsulitis. Moreover, operators should conduct handheld US- and conventional US-guided methods to determine which of the two devices is more convenient to use. A wide range of operators, from less skilled to well trained, should be surveyed in investigating the convenience of use of US devices and the operator satisfaction in using each device We checked whether participants were doing the prescribed exercise properly during outpatient visits but did not check the compliance by quantifying it. This could be a bias in treatment efficacy.

\section{Conclusion}

The results of this study showed no statistical differences in treatment efficacy between the combined use of handheld US and FL and the use of conventional US alone during shoulder IA corticosteroid injection. Furthermore, the combined use of US and FL can increase the accuracy of injection compared with conventional US alone.

\section{Acknowledgments}

The authors have not received any benefits from commercial sources for the work reported in the manuscript. This research was supported by the Technology Innovation Program (Industrial Strategic Technology Development Program-20009571, Developing AI ultrasound system and platform for intelligent intervention procedures) funded by the Ministry of Trade, Industry \& Energy (MOTIE, Korea), and the Korea Medical Device Development Fund grant funded by the Korean government (the Ministry of Science and ICT, the Ministry of Trade, Industry and Energy, the Ministry of Health \& Welfare, the Ministry of Food and Drug Safety) (Project Numbers: 9991006834, KMDF_PR_20200901_0164 and 9991007387, KMDF_PR_20200901_0170), and the Seoul R\&D Program (BT190153) through the Research and Development for Regional Industry. 


\section{Conflict of interest}

None to report.

\section{References}

[1] Prestgaard T, Wormgoor ME, Haugen S, Harstad H, Mowinckel P, Brox JI. Ultrasound-guided intra-articular and rotator interval corticosteroid injections in adhesive capsulitis of the shoulder: a double-blind, sham-controlled randomized study. Pain. 2015; 156(9): 1683-91.

[2] Manske RC, Prohaska D. Diagnosis and management of adhesive capsulitis. Curr Rev Musculoskelet Med. 2008; 1(3-4): 180-9.

[3] Le HV, Lee SJ, Nazarian A, Rodriguez EK. Adhesive capsulitis of the shoulder: review of pathophysiology and current clinical treatments. Shoulder Elbow. 2017; 9(2): 75-84

[4] Hettrich CM, DiCarlo EF, Faryniarz D, Vadasdi KB, Williams $\mathrm{R}$, Hannafin JA. The effect of myofibroblasts and corticosteroid injections in adhesive capsulitis. J Shoulder Elbow Surg. 2016; 25(8): 1274-9.

[5] Lorbach O, Anagnostakos K, Scherf C, Seil R, Kohn D, Pape D. Nonoperative management of adhesive capsulitis of the shoulder: oral cortisone application versus intra-articular cortisone injections. J Shoulder Elbow Surg. 2010; 19(2): 172-9.

[6] Bulgen DY, Binder AI, Hazleman BL, Dutton J, Roberts S Frozen shoulder: prospective clinical study with an evaluation of three treatment regimens. Ann Rheum Dis. 1984; 43(3): 353-60.

[7] van der Windt DA, Koes BW, Deville W, Boeke AJ, de Jong BA, Bouter LM. Effectiveness of corticosteroid injections versus physiotherapy for treatment of painful stiff shoulder in primary care: randomised trial. BMJ. 1998; 317(7168): 1292-6.

[8] Park KD, Nam HS, Kim TK, Kang SH, Lim MH, Park Y. Comparison of sono-guided capsular distension with fluoroscopically capsular distension in adhesive capsulitis of shoulder. Ann Rehabil Med. 2012; 36(1): 88-97.

[9] Lee HJ, Lim KB, Kim DY, Lee KT. Randomized controlled trial for efficacy of intra-articular injection for adhesive capsulitis: ultrasonography-guided versus blind technique. Arch Phys Med Rehabil. 2009; 90(12): 1997-2002.

[10] Raeissadat SA, Rayegani SM, Langroudi TF, Khoiniha M. Comparing the accuracy and efficacy of ultrasound-guided versus blind injections of steroid in the glenohumeral joint in patients with shoulder adhesive capsulitis. Clin Rheumatol. 2017; 36(4): 933-40.

[11] Patel DN, Nayyar S, Hasan S, Khatib O, Sidash S, Jazrawi LM. Comparison of ultrasound-guided versus blind glenohumeral injections: a cadaveric study. J Shoulder Elbow Surg. 2012; 21(12): 1664-8.

[12] Park KD, Nam HS, Lee JK, Kim YJ, Park Y. Treatment effects of ultrasound-guided capsular distension with hyaluronic acid in adhesive capsulitis of the shoulder. Arch Phys Med Rehabil. 2013; 94(2): 264-70.

[13] Wakeling C, Bateman A, Hatrick A, Chatakondu S. Combined fluoroscopic and ultrasound guided cervical nerve root injections. Int Orthop. 2016; 40(12): 2547-51.

[14] Stone J, Matchett G. Combined ultrasound and fluoroscopic guidance for radiofrequency ablation of the obturator nerve for intractable cancer-associated hip pain. Pain Physician. 2014; 17: E83-7.
[15] Khan JS, Krane EJ, Higgs M, Pritzlaff S, Hoffinger S, Ottestad E. A case report of combined ultrasound and fluoroscopicguided percutaneous radiofrequency lesioning of the obturator and femoral articular branches in the treatment of persistent hip pain in a pediatric patient. Pain Pract. 2019; 19(1): 52-6.

[16] Tse KH, Luk WH, Lam MC. Pocket-sized versus standard ultrasound machines in abdominal imaging. Singapore Med J. 2014; 55(6): 325-33.

[17] Greaves K, Jeetley P, Hickman M, Dwivedi G, Sabharwal N, Lim T, et al. The use of hand-carried ultrasound in the hospital setting - a cost-effective analysis. J Am Soc Echocardiogr. 2005; 18(6): 620-5.

[18] Vierola H. Ultrasonography-guided contrast media injection toshoulder joint using a posterior approach: a technique worth tryingActa. Radiol. 2004; 45: 616-7.

[19] Lundberg BG. The frozen shoulder. Clinical and radiographical observations. The effect of manipulation under general anesthesia. Structure and glycosaminoglycan content of the joint capsule. Local bone metabolism. Acta Orthop Scand Suppl. 1969; 119: 1-59.

[20] van Holsbeeck MT, Introcaso JH. Musculoskeletal Ultrasound. 2nd edn. Philadelphia: Mosby, 2001.

[21] Steinbrocker O, Argyros TG. Frozen shoulder: treatment by local injection of depot corticosteroid. Arch Phys Med Rehabil. 1974; 55(5): 209-13.

[22] Kieser M, Wassmer G. On the use of the upper confidence limit for the variance from a pilot sample for sample size determination. Biom J. 1996; 38(8): 941-9.

[23] Gordon JA, Breitbart E, Austin DC, Photopoulos CD, Kelly JD. Adhesive capsulitis: diagnosis, etiology, and treatment strategies. In: Kelly JD IV, ed. Elite Techniques in Shoulder Arthroscopy: New Frontiers in Shoulder Preservation. Cham: Springer International Publishing, 2016; 149-68.

[24] Lo SF, Chu SW, Muo CH, Meng NH, Chou LW, Huang WC, et al. Diabetes mellitus and accompanying hyperlipidemia are independent risk factors for adhesive capsulitis: a nationwide population-based cohort study (version 2). Rheumatol Int. 2014; 34: 67-74.

[25] McAlister I, Sems SA. Arthrofibrosis after periarticular fracture fixation. Orthop Clin N Am. 2016; 47(2): 345-55.

[26] Jones A, Regan M, Ledingham J, Pattrick M, Manhire A, Doherty M. Importance of placement of intra-articular steroid injections. BMJ. 1993; 307(6915): 1329-30.

[27] Eustace JA, Brophy DP, Gibney RP, Bresnihan B, FitzGerald O. Comparison of the accuracy of steroid placement with clinical outcome in patients with shoulder symptoms. Ann Rheum Dis. 1997; 56(1): 59-63.

[28] Narouze SN. Ultrasound-guided cervical spine injections: ultrasound "prevents" whereas contrast fluoroscopy "detects" intravascular injections. Reg Anesth Pain Med. 2012; 37(2): 127-30.

[29] Khan JS, Krane EJ, Higgs M, Pritzlaff S, Hoffinger S, Ottestad E. A case report of combined ultrasound and fluoroscopicguided percutaneous radiofrequency lesioning of the obturator and femoral articular branches in the treatment of persistent hip pain in a pediatric patient. Pain Pract. 2019; 19(1): 52-6.

[30] Chaiban G, Paradis T, Atallah J. Use of ultrasound and fluoroscopy guidance in percutaneous radiofrequency lesioning of the sensory branches of the femoral and obturator nerves. Pain Pract. 2014; 14(4): 343-5.

[31] Stone J, Matchett G. Combined ultrasound and fluoroscopic guidance for radiofrequency ablation of the obturator nerve for intractable cancer-associated hip pain. Pain Physician. 2014; 17(1): E83-7. 
[32] Wakeling C, Bateman A, Hatrick A, Chatakondu S. Combined fluoroscopic and ultrasound guided cervical nerve root injections. Int Orthop. 2016; 40(12): 2547-51.

[33] Gaujoux-Viala C, Dougados M, Gossec L. Efficacy and safety of steroid injections for shoulder and elbow tendonitis: a metaanalysis of randomised controlled trials. Ann Rheum Dis. 2009; 68: 1843-1849.
[34] Wakeling C, Bateman A, Hatrick A, Chatakondu S. Combined fluoroscopic and ultrasound guided cervical nerve root injections. Int Orthop. 2016; 40(12): 2547-51.

[35] Stone J, Matchett G. Combined ultrasound and fluoroscopic guidance for radiofrequency ablation of the obturator nerve for intractable cancer-associated hip pain. Pain Physician. 2014; 17(1): E83-7. 